\title{
Influence of plasma edge dynamics on blob properties in the stellarator TJ-K
}

\author{
G. Fuchert ${ }^{1}$, G. Birkenmeier ${ }^{2}$, B. Nold $^{1}$, M. Ramisch ${ }^{1}$, U. \\ Stroth $^{2}$ \\ ${ }^{1}$ Institut für Grenzflächenverfahrenstechnik und Plasmatechnologie, University \\ of Stuttgart, 70569 Stuttgart, Germany \\ 2 Max-Planck-Institut für Plasmaphysik, EURATOM Association, 85748 \\ Garching, Germany \\ E-mail: fuchert@igvp.uni-stuttgart.de
}

\begin{abstract}
.
Blob properties are studied in the scrape-off layer of the stellarator TJ-K. Langmuir probes and a fast camera are used to investigate the generation rate as well as the blob size and velocity scaling. Discharges with different ion species and magnetic field strengths provide access to a large range of plasma parameters. It was found that almost every large amplitude drift-wave in the edge of the confined plasma triggers blob generation in the scrape-off layer, which implies that the birth rate of blobs is determined by the turbulence in the edge. Furthermore, the cross-field size of the blobs seems to be correlated with the size of the generating drift waves. Since the observed radial propagation velocity of the blobs is well described by a size dependent blob velocity model, the size coupling between drift waves and blobs has also impact on the blob velocities. Thus, the presented results imply that the dynamics in the edge have a large influence on the blob properties in the scrape-off layer.
\end{abstract}

PACS numbers: 52.25.Xz,52.30.-q,52.35.Ra,52.55.Hc,52.70.Kz 


\section{Introduction}

Large density structures elongated along the magnetic field lines are observed in many different magnetized plasmas (see Ref. [1] and references therein) including, but not limited to, fusion plasmas of tokamaks and stellarators. Those structures, often referred to as blobs, contribute significantly to the particle and energy transport in the scrape-off layer (SOL) of toroidally confined plasmas 2. Thus, understanding of generation and dynamics of blobs is needed to predict the particle and energy flux to the walls of a future fusion reactor. Although understanding of blob generation [3, 4, 5, 6] and evolution [1, 7, 8, has improved a lot over the recent years, quantitative aspects of blob properties (like the generation rate and blob sizes) are still not resolved completely. Blobs contribute to the SOL transport because of their radial outward propagation. The particle transport due to blobs can be estimated by the contribution of a single blob times the so-called packing fraction $f_{\mathrm{p}}[9]$ :

$$
\Gamma \propto n_{\mathrm{b}} \cdot v_{r, \mathrm{~b}} \cdot f_{\mathrm{p}} .
$$

$n_{\mathrm{b}}$ is the average density in a blob and $v_{r, \mathrm{~b}}$ its outward propagation velocity while $f_{\mathrm{p}}$ depends on $v_{r, \mathrm{~b}}$. the blob size $\delta_{\mathrm{b}}$ and the waiting time of subsequent blobs $t_{\mathrm{w}}$ : $f_{\mathrm{p}}=2 \delta_{\mathrm{b}} / v_{r, \mathrm{~b}} t_{\mathrm{w}}$ [1, 10. This simple model already illustrates which blob properties influence directly the transport caused by blobs. Consequently, knowledge about what is determining $n_{\mathrm{b}}, v_{r, \mathrm{~b}}, \delta_{\mathrm{b}}$ and $t_{\mathrm{w}}$ is essential to predict and possibly control the blob transport. This introduction summarizes very briefly how well the different properties are understood today.

Only a few studies concerning the blob density $n_{b}$ exist. There are indications that $n_{\mathrm{b}}$ is determined mainly by the background density in the birth region of blobs [9]. The radial propagation velocity of blobs has been addressed in a vast amount of theoretical and experimental work: The dynamics of blobs has been described by a 2D model [8, 11, regarding the blob as a filament with a parallel mode number of zero $\left(k_{\|}=0\right)$, which is polarized by "effective gravity" like, e. g., magnetic curvature. The blob polarization causes poloidal electric fields and, hence, radial $\mathbf{E} \times \mathbf{B}$ drifts. Analytically, the evolution of an isolated blob filament in a simple magnetized torus with homogeneous background plasma can be described by the continuity equation of the density together with the vorticity equation 8

$$
\frac{2 T_{e}}{R B} \frac{\partial n}{\partial y}-\frac{n m_{i}}{B^{2}}\left(\frac{\partial}{\partial t}+\mathbf{v}_{E \times B} \cdot \nabla\right) \nabla^{2} \phi=D(\phi),
$$

where $y$ denotes the poloidal direction, $T_{e}$ the electron temperature, $R$ the major radius, $B$ the magnetic field strength, $n$ the plasma density, and $\phi$ the plasma potential. $D(\phi)$ corresponds to different closures describing blob damping mechanisms. To derive Eq. 22 it is assumed that the ions are cold $\left(T_{i} \ll T_{e}\right)$ and no temperature fluctuations are connected to the blob $\left(\tilde{T}_{e, i}=0\right)$. For cold plasmas this is often a good approximation while in hotter fusion plasmas the dynamics will differ from this prediction. One possible closure $D(\phi)$ considering the effect of parallel currents in sheath-limited blob filaments and collisions of ions with neutrals is treated in Refs. [12] and [13. The resulting approximate solution for the blob velocity is given by

$$
v_{r, \mathrm{~b}}=\frac{\sqrt{\frac{2 \delta_{\mathrm{b}}}{R}} c_{s}}{1+\frac{1}{\rho_{s}^{2} l_{\|}} \sqrt{\frac{R}{2}} \delta_{\mathrm{b}}^{5 / 2}+\frac{\nu_{\mathrm{in}} \sqrt{R \delta_{\mathrm{b}}}}{\sqrt{2} c_{s}}} \frac{\delta n}{n_{0}},
$$


with the sound velocity $c_{s}$, neutral ion collision frequency $\nu_{\text {in }}, \delta n$ is the peak density in the blob reduced by the background density $n_{0}$, and $l_{\|}$the parallel length of the blob (e.g. the distance between two limiter plates). An agreement with this predicted velocity has been found in TORPEX [12. It is interesting to note that in this model the velocity depends on the blob size.

There are predictions from the 2D models mentioned above where characteristic size scalings are inferred from stability considerations against secondary instabilities (see Ref. 1] and references therein). The results depend on the closure $D(\phi)$ in Eq. (2). If only parallel currents to the limiters are considered as damping mechanism, the size of the most stable blobs is given [14] by

$$
\delta_{*}=2 \rho_{s} \cdot\left(l_{\|} / \rho_{s} R\right)^{1 / 5} \propto \rho_{s}^{4 / 5},
$$

with $\rho_{s}=\sqrt{m_{i} T_{e}} / e B$. Equation (4) relates the size of the most stable blobs to the drift scale $\rho_{s}$. However, since the generation process of blobs is not yet fully understood, there is no theoretical prediction with which cross-field size blobs are generated.

For the same reason it remains unclear what determines the generation rate of blobs. There are experimental indications pointing to a coupling of turbulent fluctuations in the confined plasma and the occurrence of blobs in the SOL. Experiments at Alcator C-Mod [15] showed that the birth rate of blobs is connected to the typical drift-wave frequency. In the linear device PISCES, it was observed that large amplitude density fluctuations in the source free region are correlated with density bursts in the main plasma column. In both regions, the waiting-time distribution (WTD) was investigated, i. e. the distribution of time durations between two subsequent events of the same type. The WTDs have been found to be comparable in both regions, which points to a direct coupling between turbulent fluctuations in the main plasma and blobs in the source free region of PISCES [16.

In this paper, studies on the generation rate as well as the blob size and velocity scaling at the stellarator TJ-K are presented. The aim of these studies is to understand what determines those properties, since their understanding is essential for the prediction of the blob induced transport. After a short introduction to TJ$\mathrm{K}$ in Sec. 2 and to the applied data analysis methods in Sec. 3 the experimental results are presented in Sec. 4. A qualitative introduction to the dynamics of blobs in TJ-K is given together with a comparison of the probe and camera data. Then the generation rate is analyzed and the connection to the edge turbulence is investigated. The blob size and its scaling with the drift scale $\rho_{s}$ are analyzed for different plasma parameters. Finally, the blob velocity in TJ-K is determined and compared to theoretical predictions. The results are discussed in Sec. 5 .

\section{Experimental setup}

The presented measurements were performed at the stellarator TJ-K [17, 18, in hydrogen, deuterium, helium, neon and argon discharges with low $(70 \mathrm{mT})$ and high $(300 \mathrm{mT})$ magnetic field in order to vary the plasma parameters over a broad range. All discharges were heated with microwaves at 2.45 and $8 \mathrm{GHz}$ for the low field and high field, respectively [19]. The electron density $n_{e}$ is around $10^{18} \mathrm{~m}^{-3}$ and the electron temperature $T_{e}$ is about $10 \mathrm{eV}$. The plasma was limited by two poloidal limiter plates, which were introduced to enlarge the scrape-off layer (SOL) with constant connections lengths. The blob properties were studied with Langmuir probes and a high-speed 


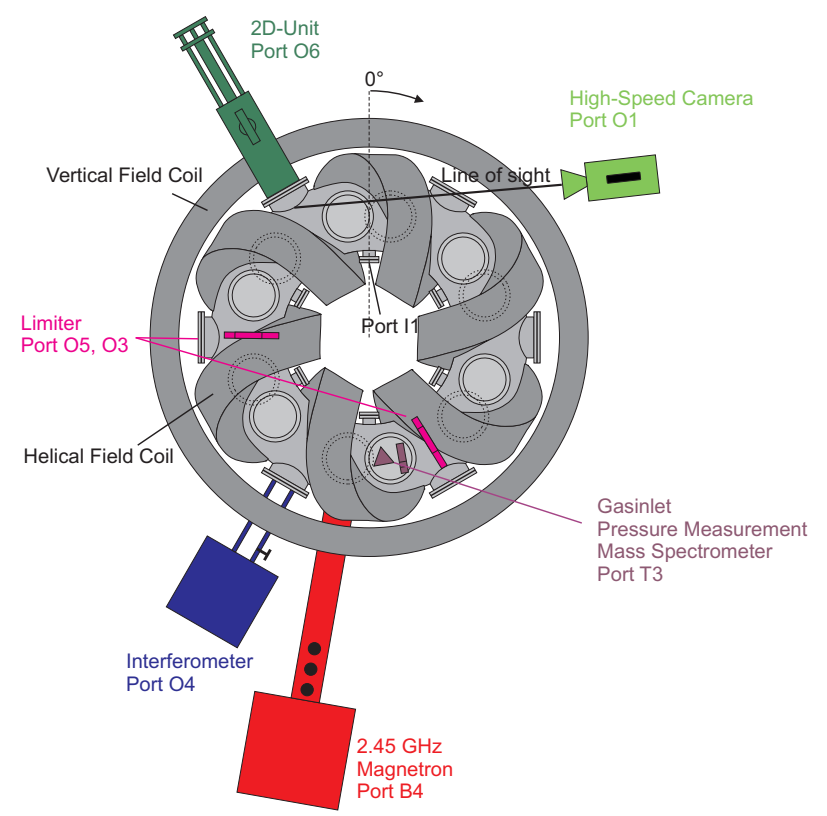

Figure 1. Experimental setup at TJ-K with two limiter plates and the line of sight of the fast camera.

camera. A 2D movable probe measured floating potential and ion-saturation current over a whole cross section of the torus with a step size of $1 \mathrm{~cm}$ in both directions. An additional fixed reference probe was used as trigger source for conditional averaging (see Sec.3.1). Temperature fluctuations are negligible in TJ-K [20, since the $T_{e}$-profile is relatively flat. Therefore, $2 \mathrm{D}$ fluctuation data from plasma potential and density can be deduced from the data. The camera measures the visible light emission, which depends on the electron temperature and density. Thus, it is a measure for density fluctuations as well, as will be shown in Sec. 4.1. The camera records the entire visible spectrum without optical filters. In contrast to the probe measurement, the 2D fluctuation data is obtained instantaneously over the whole cross section, which allows for a proper statistical treatment of the investigated quantities.

The high-speed camera used is a Photron SA-5 with a frame rate up to $750 \mathrm{kfps}$ (kilo frames per second). The camera view was almost tangential to the plasma column (see Fig. 1). A deviation of about $10^{\circ}$ from the tangent results from geometrical constraints of the main field coils and the available viewing ports. Due to the low temperature of the plasmas in TJ-K, visual light is emitted by the whole plasma. This allows for measurements in the confinement region and the SOL without neutral gas injection. In order to minimize effects due to the integration along the line of sight, the depth of focus has to be small. In order to achieve this, a camera lens with focal length $f=50 \mathrm{~mm}$ and the aperture ratio $D / f=0.75$ (D is the effective aperture) was used. 


\section{Data analysis}

\subsection{Conditional averaging}

The conditional averaging technique is used to study the evolution of coherent structures [21]. In a reference signal $\tilde{x}(t)$, with a sampling interval of $\Delta t$, large fluctuation amplitudes are detected as trigger events $\left(\tilde{x}\left(t_{i}\right) \geq x_{0}>\tilde{x}\left(t_{i}-\Delta t\right)\right)$ at times $t_{i}$. A time window $\tau \in[-T / 2, T / 2]$ of fixed length $T$ is stored from another signal $\tilde{y}(t)$ around each trigger event $t_{i}$. The average over all subsequences is calculated for each time lag $\tau$ resulting in the conditional average $(\mathrm{CA}) y_{\mathrm{c}}(\tau)$ of the signal $\tilde{y}(t)=y_{\mathrm{c}}(t)+y_{\mathrm{n}}(t)$ as the coherent contribution while incoherent noise $y_{\mathrm{n}}(t)$ vanishes. With the CA technique, 2D resolved measurements are possible by using a fixed reference probe and a movable probe, which scans a poloidal cross section of the plasma 22. By combining the CAs for every probe position, the 2D time resolved $\mathrm{CA}$ is obtained. The reference probe was located in the lower outboard side in the SOL region, where the camera detects the majority of blob events [23. Usually blobs are detected using an ion-saturation current $\left(I_{\mathrm{i}, \mathrm{sat}}\right)$ signal as a reference. In TJ-K a high correlation (typically larger than 0.6 ) is observed between the floating potential $\phi_{f}$ and $I_{\mathrm{i}, \mathrm{sat}}$, leading to comparable dynamics in the CA independent from the choice of the reference signal. The only difference in the SOL is a phase shift between $\phi_{f}$ and $I_{\mathrm{i}, \text { sat }}$, which does not affect the analyses presented in this paper since the blob properties are examined when the blob in the CA reaches specific locations and not necessarily at $\tau=0$. For best possible comparability with prior experiments at TJ-K (in particular Ref. 4 4) $\phi_{f}$ is taken as reference signal and a threshold $\phi_{0}$ for the CA of two times the standard deviation was chosen.

\subsection{Size and velocity of density structures}

From time-resolved 2D density fluctuation data, structure sizes and velocities can be obtained. The detection of the structures and the methods to obtain sizes and velocities are described in the following. In a first step, local amplitude maxima are detected in the 2D data for every time step. Any of those maxima, which exceed a certain threshold (e.g. two times the standard deviation), is associated with coherent structures in the fluctuation data. All surrounding measurement positions with a fluctuation amplitude of at least half the local blob maximum are considered to show the same structure. The detection of false positives due to noise can be prevented by using a size threshold of a few times the spatial resolution of the measurement. In the next step, an ellipse is fitted to the density structure and from the geometrical parameters of the fit the size is determined. The velocity can be measured by tracking the center of the ellipse over several time steps. This method is applied to both the camera data directly and the 2D CA results in the ion-saturation current of the probes. The procedure of fitting an ellipse to the data is done by moments analysis 24 . The CA result of the probe data allows for tracking a single average event, only. I. e., applied to the probe data, the method lacks in information on the statistical distribution of structure properties. In contrast, the 2D camera measurements capture the dynamics of many single events, which enables a statistical treatment. However, since the camera measurements are affected by light integration along the line of sight, one has to be cautious with the interpretation of the obtained structure size. In Fig. 2 intensity fluctuations during a blob event are shown together with an ellipse fitted to the data. The dashed line represents the projection of a field line segment 


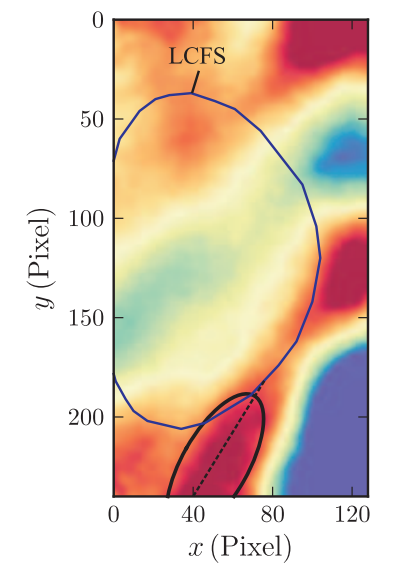

Figure 2. Intensity variations during a blob event with an ellipse fitted to the blob. The solid line represent the LCFS in the focused plane, the dashed line is the projection of a field line through the blob. The major axis of the ellipse is oriented parallel to the field line due to the extension of the blob filament along the field and the light integration along the line of sight.

crossing the blob. The major axis of the fitted ellipse is oriented parallel to this field line segment. This suggests that the extension of the blob filament parallel to the magnetic field influences the length of the major axis. Thus, the minor axis is used as a representative of the cross-field blob size. For further analysis, only isolated structures are used, which are completely located either in the confinement region or in the SOL without overlap. In the analyses presented in this paper only structures in the lower part of the edge/SOL (as in Fig. 2) are taken into account where the size obtained from the camera measures the poloidal size. This has been cross-checked with the probe measurements.

\section{Results}

\subsection{Conditional average of the image and probe data}

In Ref. 4], the generation and dynamics of blobs have been studied with Langmuir probes. In the present section, the comparability of probe and camera measurements for similar experiments is examined. Figure 3 a) shows an image series of a propagating structure as obtained by conditionally averaging fluctuations in the ion-saturation current. The dot in the lower right corner indicates the position of the reference probe. In the confined plasma of TJ-K, drift-wave turbulence is accompanied by coherent structures with lifetimes in the range of $10-100 \mu \mathrm{s}$ [25]. These structures propagate into the electron diamagnetic direction (clockwise in the shown figures). Such a structure with a poloidal mode number of $m=3$ can be seen in Fig. (3 a) at $\tau=-0.02 \mathrm{~ms}$. The density structure extends partly over the last closed flux surface (LCFS, depicted as solid line) into the SOL. As shown in Ref. 4], the part of the structure extending into the SOL can grow when a dipole structure appears in the turbulent potential fluctuations and density is advected outwards by the resulting $\mathbf{E} \times \mathbf{B}$-drift. Following the next few $\mu$ s in Fig. [3a), one can see the structure near the reference propagate and grow. It detaches from the bulk and forms a blob. Fig. 3 b) 
a)

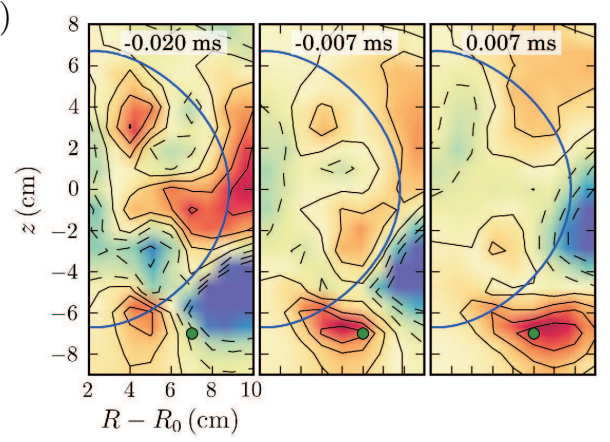

b)

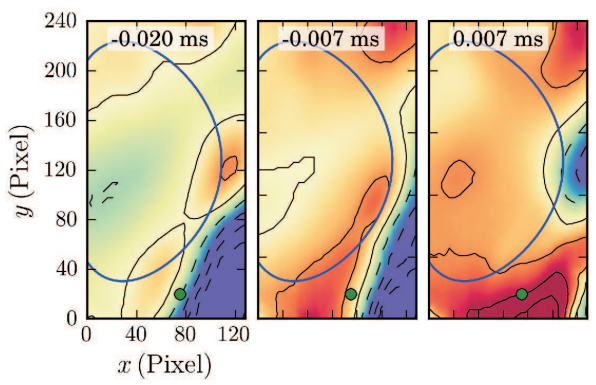

Figure 3. Conditional average of density fluctuations from probes (a) and camera (b) during blob events in a deuterium plasma. The solid line depicts the LCFS, the dot (green) is the location of the reference probe. Positive fluctuations are shown as solid contour lines (red) and negative ones as dashed contour lines (blue).

a)

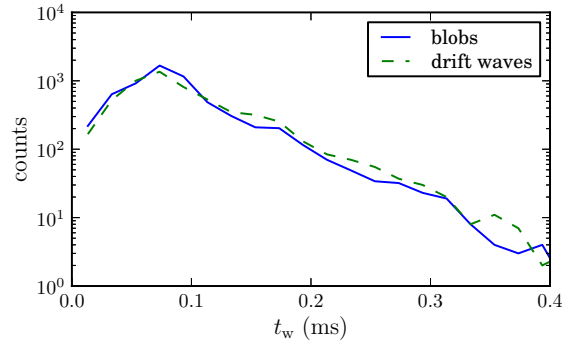

b)

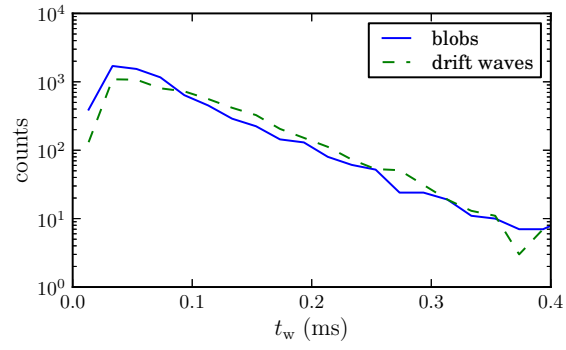

Figure 4. Comparison of waiting time distributions for blobs and drift waves in a hydrogen (a) and helium (b) discharge obtained from high-speed imaging.

shows the CA of intensity variations from the image data of the fast camera for the same discharge. A pixel in the region where the blobs are observed is used as reference signal (dot). Comparing Fig (3)a) and b) it can be seen that the intensity variations are similar to the density fluctuations. In the confinement region, the mode structure can be seen in the first image of Fig $3 \mathrm{~b}$ ), but it is blurred due to the integration along the line of sight. In the SOL, where the blob properties are studied, the agreement is much better. This can be seen by comparing the structures in the top and bottom right for both diagnostics at $\tau=0.007 \mathrm{~ms}$. When the image data is cross correlated with ion-saturation current measurements large correlation values up to 0.8 are obtained, which provides further evidence that the camera observes density fluctuations.

\subsection{Birth rate of blobs}

On the conditional average, drift waves in the edge appear to be involved in the blob generation. This is consistent with previous results in TJ-K [4]. Note that in the course of this paper the term edge refers only to the edge of the confined plasma to distinguish this region from the SOL. From the CA, however, it cannot be concluded whether each single blob is related to a drift wave or with what probability an existing drift wave triggers blob ejection. To elucidate this connection, the waiting-time distribution (WTD) and generation rate is compared for drift waves in the edge and blobs in the SOL. 


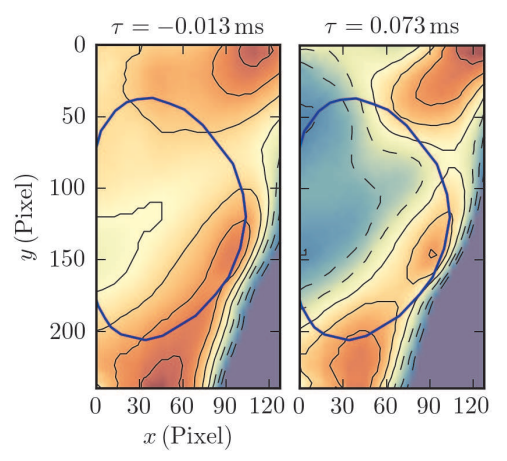

Figure 5. The CA of the camera data reveals a comparable density pattern in the shown hydrogen discharge after $\Delta \tau \approx-90 \mu \mathrm{s}$. Both images are scaled to their maximum intensity for better visualization.

The WTD for blobs and large drift waves can be obtained from the image data by the structure detection described in section 3.2. The occurrence time $t_{i}$ of structure $i$ is defined as the time when the intensity amplitude in a small predefined area of the images reaches its maximum during an ongoing event. The waiting time between two subsequent events is defined as $t_{\mathrm{w}}=t_{i+1}-t_{i}$. Figure 4 shows the WTDs of blobs and drift waves in low-field hydrogen (left) and helium (right) discharges. For a purely random blob generation process, an exponential decay of the WTD would be expected 27. Such a behaviour has been found at Alcator C-Mod 28. In TJ-K, an exponential decay is found for $t_{\mathrm{w}}>100 \mu \mathrm{s}$. However, there is a clear peak in the WTDs at about $t_{\mathrm{w}}=90 \mu \mathrm{s}(\mathrm{H})$ and $76 \mu \mathrm{s}(\mathrm{He})$ for both, drift waves and blobs. This feature varies scarcely for discharges with comparable plasma parameters, but is located at different times for the various gases used in TJ-K. For the drift waves in the confined plasma this peak can be understood as follows: If the typical lifetime of the turbulent structures is longer than the time $T_{\mathrm{t}}$ for a complete poloidal transit divided by the poloidal mode number $m$, the density pattern inside the LCFS recurs after $T_{\mathrm{t}} / m$ due to the poloidal rotation of the drift wave. This is indeed the case as can be seen from Fig. 5 . It shows two images with a time distance of $\Delta \tau \approx 90 \mu$ s of the conditionally averaged camera data of a hydrogen discharge. Both images show a comparable density pattern in the confined plasma and in the SOL. Experimental values for the dominant poloidal mode number $m$ between three and four, poloidal velocities of the drift waves $\leq 2 \mathrm{~km} / \mathrm{s}$ and a poloidal circumference near the LCFS of $U_{\text {pol }} \approx 50 \mathrm{~cm}$ yield values for $T_{\mathrm{t}} / \mathrm{m}$ in the range of 50 to $100 \mu \mathrm{s}$, which is in good agreement with the observed peaks in the WTDs. Taking into account the generation mechanism presented in Sec. 4.1, it becomes clear that the peak in the WTD for the SOL events is caused by blobs generated at the same location with a time distance of $T_{\mathrm{t}} / m$ by separate density maxima of a poloidally rotating drift wave. This connection is also apparent in the power spectra of $I_{\mathrm{i}, \mathrm{sat}}$ measurements in the edge and the SOL. Figure 6 shows such spectra for the hydrogen plasma analyzed above. In both spectra a peak is observed at the inverse of the peak time in the WTD. In the edge this peak corresponds to the quasi-coherent modes with lifetimes of about $100 \mu \mathrm{s}$ arising from drift-wave turbulence [25]. Due to blob generation by density maxima of these quasicoherent modes, a peak at the same frequency is present in the SOL power spectrum. 


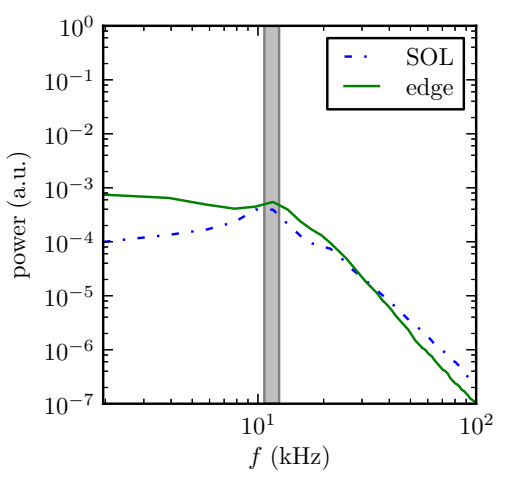

Figure 6. Power spectra of $I_{\mathrm{i}, \mathrm{sat}}$ measurements in the edge (dashed line) and the SOL (solid line) of a hydrogen plasma. The area shaded in gray marks the inverse of the peak in the WTD $t_{\mathrm{w} \text {,peak }} \pm t_{\mathrm{ex}}$ where $t_{\mathrm{ex}}$ is the camera exposure time that sets the accuracy of $t_{\mathrm{w}, \text { peak }}$.

But not only the shape of both WTDs shows a good agreement, also the total number of detected events is similar, as can be seen from Fig. 4. The detection rates for blobs with intensity amplitudes larger than the standard deviation and density maxima of drift waves are about $10,000 / \mathrm{s}$ for the analyzed discharges. This corresponds to the typical frequency range of a few $\mathrm{kHz}$ for the drift-wave turbulence in TJ-K, which reflects that blobs principally appear in conjunction with drift waves. Thus, it can be concluded that the generation rate of blobs in the SOL is determined by properties of the drift-wave turbulence in the edge plasma. The results presented here are in agreement with probe measurements in comparable TJ-K discharges, where the peak of the WTD for intermittent structures in the SOL was found close to the drift-wave period [26].

\subsection{Blob-size scaling}

This section investigates the scaling of the poloidal structure size $\delta$ of blobs in the SOL with the drift scale $\rho_{s}$. The results are compared to the prediction of Eq. (4) and to the size scaling for coherent structures in the confinement region of TJ-K to study if there is a relation between the size of the blob and the generating drift wave. For drift-wave turbulence, a scaling $\delta \propto \rho_{s}^{\alpha}$ with $\alpha \approx 0.5$ was found [25]. However, there were indications that $\alpha$ decreases if the structure sizes get too close to the system size, which might be the case for the limited discharges presented in this paper. Here, $\rho_{s}$ is varied by using different gases (hydrogen, deuterium, helium, neon, and argon) and two different magnetic field strengths. Additionally, for low-field hydrogen and helium discharges, shots with different heating power and thus electron temperature, were analyzed. The results of the blob size measurements are shown in Fig. 7 a). The scaling exponents are $\alpha_{\text {cam }}=0.19 \pm 0.03$ and $\alpha_{\text {probe }}=0.26 \pm 0.07$. The errors for the probe data arise from systematic inaccuracies in the blob sizes of half the grid spacing. If the same analysis is repeated for the coherent structures in the confined plasma, the scaling laws with $\alpha_{\text {cam }}=0.23 \pm 0.04$ and $\alpha_{\text {probe }}=0.21 \pm 0.11$ are obtained. All exponents are comparable within the error bars. The scaling of the blob size is weaker than predicted for the most stable blobs according to Eq. (4). Note, however, that the 

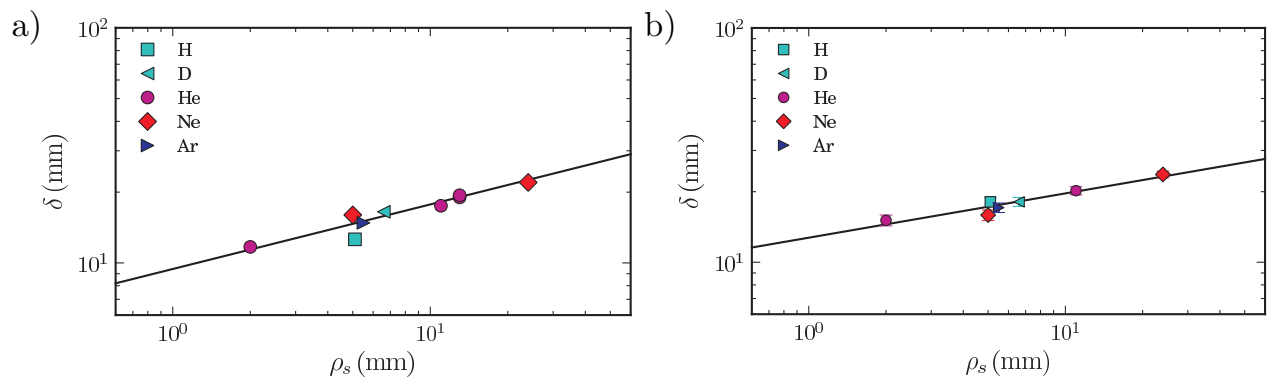

Figure 7. $\rho_{s}$-scaling of the poloidal blob size as measured with probes (a) and fast camera (b). The error bars for the camera measurement are in the range of the symbol size. Since the CA technique is used to determine the size for the probe data, no statistical error is available for the probe measurements.

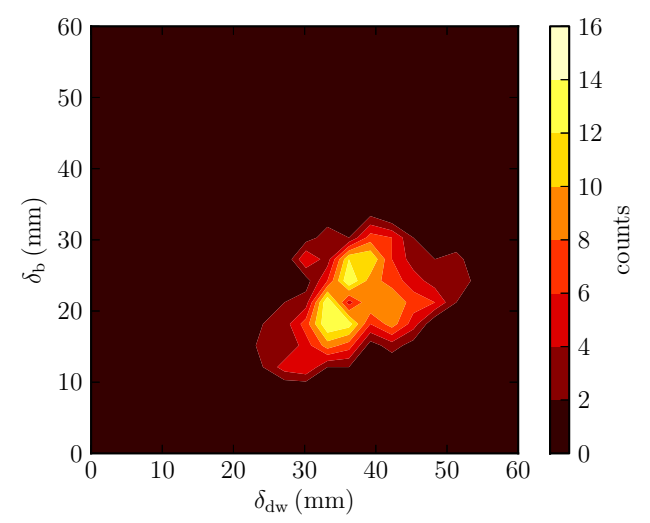

Figure 8. Comparison of poloidal sizes of blobs detected in the camera data and of coherent structures inside the LCFS right before the blob ejection. The displayed data is from a neon low-field plasma.

found size scaling corresponds to blobs directly after generation and not necessarily to the most stable ones as required for the predicted $\rho_{s}$ dependence.

If blobs are generated by drift waves, it is reasonable to assume that blobs also inherit the size properties of drift waves, e. g. their scaling behaviour as observed here. This hypothesis is supported by the fact that a correlation is found in the camera data between the size of single blob events and coherent structures inside the LCFS. For every blob event detected in the data, the size of coherent structures inside the plasma right before the blob event was analyzed. Figure 8 shows a sample plot of the observed sizes for a neon discharge. For every discharge used for the scaling, a linear regression was performed separately to check if there is a correlation between the different sizes. All analyzed shots showed a weak but positive correlation of approximately 0.2 , varying between 0.14 and 0.27 for the single shots. Low correlation values can be caused by a non-linear relation or additional effects that influence the blob size simultaneously. Nevertheless, the result indicates that larger drift waves eject larger blobs. 
a)

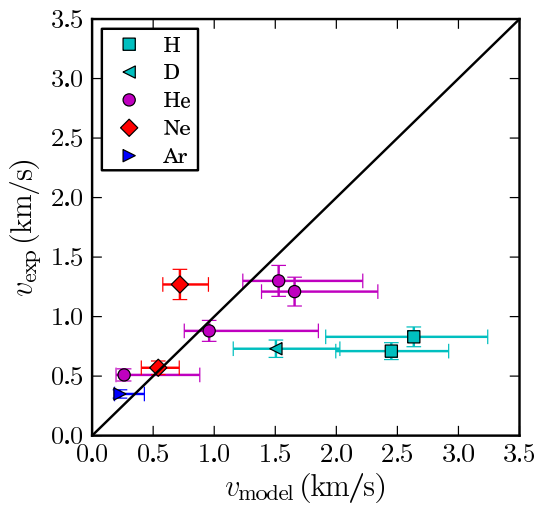

b)

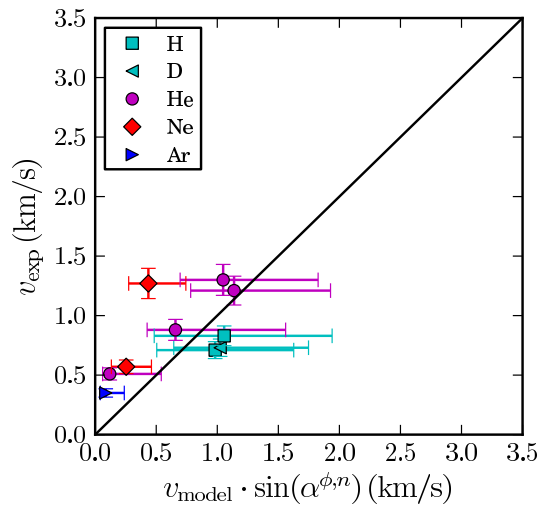

Figure 9. Comparison of measured blob velocities $\left(v_{\exp }\right)$ with a) predictions of Eq. (3) and b) the same prediction but with an empirical correction factor, describing the effect of a cross phase $<\pi / 2$.

\subsection{Velocity scaling}

In order to compare the prediction according to Eq. (3) to the blob velocities measured in TJ-K, the radial outward velocity is defined as $v_{\text {blob }}=\mathrm{d} / \mathrm{d} t d_{\mathrm{LCFS}}$, where $d_{\mathrm{LCFS}}$ is the distance of the center of the blob to the LCFS. The exact blob positions needed to determine the velocity are taken from the $\mathrm{CA}$ of the ion-saturation current measurement. Electron temperature profiles were measured for every discharge to evaluate $c_{s}$ and $\rho_{s}$ in Eq. (3). The term $\delta n / n_{0} \approx 1$ was determined from the ionsaturation current measurements and the collision frequency $\nu_{\text {in }}$ has been calculated by $\nu_{\text {in }}=n_{n} \sigma\left(k T_{i} / m_{i}\right)^{1 / 2}$ using a measured neutral pressure $n_{n}$ (about $5 \cdot 10^{18} / \mathrm{m}^{3}$ depending on the discharge), an estimated ion temperature $T_{i}$ of $1 \mathrm{eV}$ [29], and an estimated neutral-ion cross section of $\sigma=10^{-15} \mathrm{~cm}^{2}$ (which is large compared to values given in e.g. Ref. [30]). Even with the large choice for $\sigma$ the resulting collision term in Eq. (3) is smaller than 0.01 and, hence, negligible compared to the 1 in the denominator of that equation. Figure 9 a) shows a good overall agreement with the measured blob velocities, despite the fact that the model is two dimensional and, therefore, does not account for the complex stellarator geometry. The absolute values of the experimental values are in the range of the prediction. Since the variation of the ion mass and the magnetic field has large effects on the plasma parameters, additional three low-field helium discharges with different heating powers where compared. In agreement with the prediction, the resulting change in $\delta_{\mathrm{b}}$ and $T_{e}$ goes along with an increase of the blob velocity (see Fig. 9 a)). However, it is observed that the blob velocity in the hydrogen discharges (and less pronounced in deuterium) is not only significantly smaller than the prediction, but also deviates clearly from the trend observed for the other ion species. The model describes the various gases differently well. The reason for this deviation could be differences in the cross phase between density and potential $\alpha^{\Phi, n}$, which is measured to be significantly smaller for hydrogen than for the other gases. $\alpha^{\Phi, n}$ is estimated from the cross correlation of the CA of the ion saturation current and floating potential measurements [31. The blob model from Ref. [12, however, describes blobs as ideal interchange objects with a cross phase of $\pi / 2$. Any deviation from this should reduce the effectiveness of the interchange drive and, therefore, the velocity of the blobs. In the extreme case of $\alpha^{\Phi, n}=0$, the 
outward propagation would vanish. To account for this, an empirical correction factor of $\sin \left(\alpha^{\Phi, n}\right)$ is multiplied to Eq. (3).

$$
v_{\text {blob }}=\sin \left(\alpha^{\Phi, n}\right) \cdot v_{\text {model }}
$$

The comparison with the experimental data is shown in Fig. 9 b). For hydrogen and deuterium a much better agreement is obtained if the cross-phase correction is taken into account.

\section{Conclusions}

Blob properties were studied in the stellarator TJ-K using a fast camera and Langmuir probes. Prior to this work, it was known that turbulent structures extend into the SOL and transport density outwards by turbulent $\mathbf{E} \times \mathbf{B}$ drifts, forming density blobs in the SOL [4. In the experiments presented in this paper, the blob formation is not a rare process but seems to be triggered by almost every large amplitude drift wave in the edge plasma. This is reflected in the waiting-time distributions, which are similar for blobs and drift waves. The mode structure of drift waves even allows for the generation of more than one blob in a row at a fixed position by different density maxima of the same structure. This phenomenon originates from the relatively long lifetimes of the coherent structures in the edge compared to the characteristic time scales of the structure propagation and might, therefore, be quite specific for TJ-K. Nevertheless, it underlines the close connection between the occurrence of drift waves and blob generation.

Furthermore, the blob size was measured over a large range of plasma parameters and compared to the characteristic $\rho_{s}$-scaling of the cross-field sizes of turbulent structures in the confinement region of TJ-K. Even though blobs and drift waves have different dynamical properties, the found size scaling is comparable, which points to larger drift waves generating larger blobs. This hypothesis is supported by a positive correlation between the size of single blob events and the size of the generating drift wave, as was found from fast imaging.

Finally, the blob velocity was measured and compared to the theoretical blob model described in [8, 11, 12. Despite the fact that this model does not include complex magnetic field geometries as in TJ-K, a remarkable agreement with the prediction according to (3) was found. There are, however, deviations from the prediction, which could be described by the cross phase between density and potential. The cross phase differs between the ion species probably due to a different relevance of the interchange drive leading to a slower outward motion of the blobs. By including an empirical phase factor of $\sin \left(\alpha^{\phi, n}\right)$, a better agreement with the prediction is obtained. Since Eq. (3) suggests a dependence of the velocity on the blob size, the observed influence of the drift waves on the blob sizes implies that the drift waves also influence indirectly the outward velocity of the blob filaments.

In summary, it was found that the blob generation rate, the blob size perpendicular to the magnetic field and the blob radial velocity are closely related to the properties of the drift-wave turbulence in the plasma edge inside the LCFS. In the low temperature plasma of TJ-K, the generation of blobs is triggered by turbulent density fluctuations in the vicinity of the LCFS as consistent with observations in fusion experiments, e. g. by gas-puff imaging in Alcator C-Mod [32] and NSTX 9, 33, amongst others (see Ref. [1] and references therein). This points to an universal 
process behind blob generation. In this work, the transmission of turbulence properties from the edge to blob filaments in the SOL is found to be an important aspect of blob generation. This implies that pure SOL models may not be sufficient to fully understand the SOL transport. The edge dynamics has to be included to account for the coupling of the properties within the blob generation mechanism.

\section{References}

[1] D'Ippolito D A, Myra J R and Zweben S J 2011 Phys. Plasmas 1860501

[2] Boedo J A et al 2001 Phys. Plasmas 84826

[3] Furno I, Labit B, Podestà M, Fasoli A, Müller S H, Poli F M, Ricci P, Theiler C, Brunner S, Diallo A and Graves J 2008 Phys. Rev. Lett. 100055004

[4] Happel T, Greiner F, Mahdizadeh N, Nold B, Ramisch M and Stroth U 2009 Phys. Rev. Lett. 102255001

[5] Xu G S et al 2009 Nuclear Fusion 49092002

[6] Manz P, Xu M, Müller S H, Fedorczak N, Thakur S C, Yu J H and Tynan G R 2011 Phys. Rev. Lett. 107195004

[7] Zweben S J, Boedo J A, Grulke O, Hidalgo C, LaBombard B, Maqueda R J, Scarin P and Terry J L 2007 Plasma Phys. Controlled Fusion 49 S1

[8] Krasheninnikov S I, D'Ippolito D A and Myra J R 2008 J. Plasma Phys. 74679

[9] Myra J R, D'Ippolito D A, Stotler D P, Zweben S J, LeBlanc B P, Menard J E, Maqueda R J and Boedo J 2006 Phys. Plasmas 1392509

[10] Russell D A, Myra J R and D'Ippolito D A 2007 Phys. Plasmas 14102307

[11] D'Ippolito D A, Myra J R and Krasheninnikov S I 2002 Phys. Plasmas 9222

[12] Theiler C, Furno I, Ricci P, Fasoli A, Labit B, Müller S H and Plyushchev G, 2009 Phys. Rev. Lett. 10365001

[13] Theiler C, Furno I, Fasoli A, Ricci P, Labit B and Iraji D 2011 Phys. Plasmas 1855901

[14] Yu G Q and Krasheninnikov S I 2003 Phys. Plasmas 104413

[15] Krasheninnikov S I 2001 Phys. Lett. A 283368

[16] Antar G Y 2003 Phys. Plasmas 103629

[17] Krause N, Lechte C, Stober J, Stroth U, Ascasibar E, Alonso J and Niedner S 2002 Rev. Sci. Instrum. $\mathbf{7 3} 3474$

[18] Stroth U, Greiner F, Lechte C, Mahdizadeh N, Rahbarnia K and Ramisch M 2004 Phys. Plasmas 112558

[19] Köhn A et al 2013 Plasma Phys. Controlled Fusion 55014010

[20] Mahdizadeh N, Greiner F, Ramisch M, Stroth U, Guttenfelder W, Lechte C and Rahbarnia K 2005 Plasma Phys. Controlled Fusion 47569

[21] Nobach H et al 2007 Experimental Fluid Mechanics, edited by Tropea C, Foss J and Yarin A (Berlin: Springer-Verlag), pp. 1337-1398

[22] Block D, Teliban I, Greiner F and Piel A 2006 Phys. Scr. 200625

[23] Fuchert G, Boettcher T, Ramisch M and Stroth U 2011 Proc. 38th EPS Conference on Controlled Fusion and Plasma Physics (EPS, Strasbourg, 2011) p P2.120

[24] Rocha L, Velho L, Carvalho P C 2004 XVII Brazilian Symposium on Computer Graphics and Image Processing (SIBGRAPI) (IEEE Computer Society) p 354

[25] Ramisch M, Mahdizadeh N, Stroth U, Greiner F, Lechte C and Rahbarnia K 2005 Phys. Plasmas 1232504

[26] Hornung G, Nold B, Maggs J E, Morales G J, Ramisch M and Stroth U 2011 Phys. Plasmas 18 082303

[27] Garcia O E 2012 Phys. Rev. Lett. 108265001

[28] Garcia O E, Fritzner S M, Kube R, Cziegler I, LaBombard B and Terry J L 2013 Phys. Plasmas 20055901

[29] Enge S, Birkenmeier G, Manz P, Ramisch M and Stroth U 2010 Phys. Rev. Lett. 105175004

[30] Okuno K, 2007 NIFS-DATA-100.

[31] Carreras B A et al 1996 Phys. Plasmas 32664

[32] Terry J L et al 2003 Phys. Plasmas 101739

[33] Maqueda R J, Stotler D P and Zweben S J 2011 J. Nucl. Mater. 415459 